\title{
ANALYSIS OF PROGRESSIVELY CENSORED COMPETING RISKS DATA
}

\author{
Debasis Kundu ${ }^{1}$, Nandini Kannan ${ }^{2}$ and N. Balakrishnan ${ }^{3}$
}

\begin{abstract}
In several studies in Survival Analysis, the cause of failure/ death of items or individuals may be attributable to more than one cause. In this chapter, we consider the competing risks model when the data is progressively Type-II censored. We provide different techniques for the analysis of the model under the assumption of independent causes of failure and exponential lifetimes. The maximum likelihood estimators of the different parameters and the UMVUE's are obtained. In addition, the exact distributions of the different estimators are derived. We also derive the UMP and UMPU test for the equality of the failure rates of the competing risks.

We consider the Bayesian estimation using the Inverse Gamma distribution as a prior. To assess the performance of all these estimators, confidence intervals are developed using the exact, asymptotic, and bootstrap distributions. In the Bayesian context, we develop credible intervals for the parameters. The different methods are compared through a simulation study, and the analysis of a real dataset. Finally, we also provide some insight into inference under the Weibull model and dependent causes of failure.
\end{abstract}

Keywords and Phrases: Competing risks, failure rates, exponential distribution, maximum likelihood estimators, confidence intervals.

${ }^{1}$ Department of Mathematics, Indian Institute of Technology Kanpur, Pin 208016, INDIA.

2 Department of Management Science and Statistics, The University of Texas at San Antonio, 1604 N.West Loop, San Antonio, Texas, USA.

3 Department of Mathematics and Statistics, McMaster University, Hamilton, Ontario, Canada L8S 4K1 


\section{INTRODUCTION}

In medical studies or in the analysis of reliability data, the failure of individuals or items may be attributable to more than one cause or factor. These "risk factors" in some sense compete for the failure of the experimental unit. Consider the example of Hoel (1972), based on a laboratory experiment in which mice were given a dose of radiation at 6 weeks of age. The causes of death were recorded as Thymic Lymphoma, Reticulum Cell Sarcoma, or other. Another example is from a study of breast cancer patients (Boag 1949), where the cause of death was recorded as "cancer" or "other'. There are numerous examples in reliability experiments, where items may fail due to one of several causes. In traditional analyses of these datasets, the researcher is primarily interested in the distribution of lifetimes under one specific cause of failure, say cancer, and all other causes are combined and treated as censored data.

In recent years, however, models have been developed to assess the lifetimes of a specific risk in the presence of other competing risk factors. The data for these "competing risk models" consist of the failure time and an indicator variable denoting the specific cause of failure of the individual or item. The causes of failure may be assumed to be independent or dependent. In most situations, the analysis of competing risk data assumes independent causes of failure. Even though the assumption of dependence may be more realistic, there is some concern about the identifiability of the underlying model. Kalbfleisch and Prentice (1980), Crowder (2001) and several other authors have argued that without information on covariates, it is not possible using data to test the assumption of independent failure times. See Crowder (2001) and the monograph by David and Moeschberger (1978) for an exhaustive treatment of different competing risks models.

In this chapter, we will develop inference for the competing risk model under a very general censoring scheme. Censoring is inevitable in life-testing and reliability studies because the experimenter is unable to obtain complete information on lifetimes for all individuals. For example, patients in a clinical trial may withdraw from the study, or the study may have to be terminated at a pre-fixed timepoint. In industrial experiments, units may break accidentally. In many situations, however, the removal of units prior to failure is pre-planned in order to provide savings in terms of time and cost associated with testing.

The two most common censoring schemes are termed Type-I and Type-II censoring. 
Consider $n$ individuals under observation in a clinical study. In the conventional Type-I censoring scheme, the experiment continues upto a prespecified time $T$. Failures (deaths) that occur after $T$ are not observed. The termination point $T$ of the experiment is assumed to be independent of the failure times. By contrast, the conventional Type-II censoring scheme requires the experiment to continue until a prespecified number of failures $m \leq n$ occur. In this scenario, only the smallest lifetimes are observed. In Type-I censoring, the number of failures observed is random and the endpoint of the experiment is fixed, whereas in Type-II censoring the endpoint is random, while the number of failures observed is fixed. There have been numerous articles and books in the reliability and survival analysis literature, dealing with inference under Type-I and Type-II censoring for different parametric families of distributions.

One of the drawbacks to these conventional Type-I and Type-II censoring schemes outlined above is that they do not allow for removal of units at points other than the terminal point of the experiment. Cohen $(1963,1966)$ was one of the earliest to study a more general censoring scheme: Fix $m$ censoring times $T_{1}, \ldots T_{m}$. At time $T_{i}$, remove $R_{i}$ of the remaining units randomly. The experiment terminates at time $T_{m}$ with $R_{m}$ units still surviving. This is referred to as Progressive Type-I right censoring. Cohen (1963) discussed the estimation of parameters under this scheme for the parameters of the normal distribution.

In this chapter, we consider competing risk data under progressive Type-II censoring. The censoring scheme is defined as follows: Consider $n$ individuals in a study and assume that there are $K$ causes of failure which are known. At the time of each failure, one or more surviving units may be removed from the study at random. The data from a progressively Type-II censored sample is as follows:

$$
\left(X_{1: m: n}, \delta_{1}, R_{1}\right), \ldots,\left(X_{m: m: n}, \delta_{m}, R_{m}\right),
$$

where $X_{1: m: n}<\ldots<X_{m: m: n}$ denote the $m$ observed failure times, $\delta_{1}, \ldots, \delta_{m}$ denote the causes of failure, and $R_{1}, \ldots, R_{m}$ denote the number of units removed from the study at the failure times $X_{1: m: n}, \ldots, X_{m: m: n}$. Note that the complete and Type-II right censored samples are special cases of the above scheme when $R_{1}=R_{2}=\ldots=R_{m}=0$, and $R_{1}=R_{2}=\ldots=$ $R_{m-1}=0, R_{m}=n-m$, respectively. For an exhaustive list of references and further details on progressive censoring, the reader may refer to the book by Balakrishnan and Aggarwala (2000).

The main focus of this chapter is the analysis of the competing risk model when the 
data are progressively Type-II censored. In addition, we will assume the lifetimes under the competing risks have independent exponential distributions. We derive the maximum likelihood estimators (MLE) and uniformly minimum variance unbiased estimators (UMVUE) of the hazard rates. We also show that the MLE's and UMVUE's of the mean lifetime of the different causes may not always exist. We, therefore, propose the use of conditional MLE's of the mean lifetimes. The exact distributions of the MLE's and conditional MLE's are obtained, leading to the construction of confidence intervals for the different parameters. Bayes estimators and the corresponding credible regions of the different parameters using inverted gamma priors are also obtained.

The organization of the chapter is as follows. In Section 2, we describe the model and present the definitions and notation used throughout the chapter. The estimation of the different parameters are considered in Section 3, and their distributions are derived in Section 4. Bayesian analysis of the competing risk model is provided in Section 5. Results of a simulation study comparing the coverage probabilities and lengths of the different confidence and credible intervals are provided in Section 6. We illustrate the performance of these different techniques in Section 7 using a real dataset. Section 8 discusses some extensions and generalization of our results. Finally, some conclusions are drawn in Section 9.

\section{MODEL: DESCRIPTION AND NOTATION}

Without loss of generality, we assume that there are only two independent causes of failure. All the methods presented in this chapter may be easily extended to the case of $K>2$. We introduce the following notation: 
$X_{j i}$ : lifetime of the $i^{t h}$ individual under cause $j, j=1,2$

$F($.$) : Cumulative distribution function of X_{i}$

$F_{j}():$.$\quad cumulative distribution function of X_{j i}$

$\bar{F}_{j}($.$) : survival function of X_{j i}, \bar{F}_{j}()=.1-F_{j}($.

$\delta_{i}$ : $\quad$ indicator variable denoting the cause of failure of the $i^{\text {th }}$ individual

$m$ : the number of complete failures observed before termination

$x_{i: m: n}: \quad i^{\text {th }}$ observed failure time, $i=1, \ldots, \mathrm{m}$

$R_{i}$ : number of units removed at the time of the $i^{\text {th }}$ failure,

$R_{i} \geq 0, R_{1}+\ldots R_{m}+m=n$

$I_{j}: \quad=\left\{x_{i: m: n} ; \delta_{i}=j\right\}, \mathrm{j}=1,2$

$\left|I_{j}\right|: \quad$ cardinality of $I_{j}$ : we assume $\left|I_{j}\right|=n_{j}$

gamma $(\alpha, \lambda)$ : denotes the gamma random variable with density function $\frac{\lambda^{\alpha}}{\Gamma(\alpha)} x^{\alpha-1} e^{-\lambda x}$

Igamma $(\alpha, \lambda)$ : denotes the inverted gamma random variable with density function $\frac{\lambda^{\alpha}}{\Gamma(\alpha)} \frac{1}{x^{\alpha+1}} e^{-\frac{\lambda}{x}}$

$\exp (\lambda)$ denotes the exponential random variable with density function $\lambda e^{-\lambda x}$ $\operatorname{Bin}(N, p)$ : denotes the binomial random variable with probability mass function $\left(\begin{array}{c}N \\ i\end{array}\right) p^{i}(1-p)^{N-i}$.

We assume that $\left(X_{1 i}, X_{2 i}\right), i=1, \ldots, n$, are $n$ independent, identically distributed (i.i.d.) exponential random variables. Further, $X_{1 i}$ and $X_{2 i}$ are independent for all $i=1, \ldots n$ and $X_{i}=\min \left\{X_{1 i}, X_{2 i}\right\}$. We observe the sample

$$
\left(X_{1: m: n}, \delta_{1}, R_{1}\right), \ldots,\left(X_{m: m: n}, \delta_{m}, R_{m}\right)
$$

assuming all the causes of failure are known. If some of the causes are unknown, we denote 
them with $a^{(*)}$. Under that setup, the observations are denoted as follows:

$$
\begin{aligned}
& \left(X_{1: m: n}, \delta_{1}, R_{1}\right), \ldots,\left(X_{i_{1}-1: m: n}, \delta_{i_{1}-1}, R_{i_{1}-1}\right)\left(X_{i_{1}: m: n}, *, R_{i_{1}}\right) \\
& \left(X_{i_{1}+1: m: n}, \delta_{i_{1}+1}, R_{i_{1}+1}\right) \ldots,\left(X_{i_{k}-1: m: n}, \delta_{i_{k}-1}, R_{i_{k}-1}\right),\left(X_{i_{k}: m: n}, *, R_{i_{k}}\right), \\
& \left(X_{i_{k}+1: m: n}, \delta_{i_{k}+1}, R_{i_{k}+1}\right) \ldots,\left(X_{m: m: n}, \delta_{m}, R_{m}\right) .
\end{aligned}
$$

The $i_{1}^{t h}, \ldots, i_{k}^{t h}$ causes of failure are unknown. We also assume that $k$, the total number of unknown causes of failure, is fixed.

\section{ESTIMATION}

We assume that the $X_{j i}$ 's are exponential random variables with parameters $\lambda_{j}$ for $i=$ $1, \ldots, n$ and for $j=1,2$. The distribution function $F_{j}($.$) of X_{j i}$ has the following form:

$$
F_{j}(t)=1-e^{-\lambda_{j} t}
$$

for $j=1$ and 2. Since the minimum of 2 independent exponential random variables is also distributed as an exponential, the likelihood function of the observed data (2.1) is

$$
\begin{aligned}
L\left(\lambda_{1}, \lambda_{2}\right) & =n\left(n-R_{1}-1\right) \ldots\left(n-R_{1}-\ldots-R_{m-1}-m+1\right) \\
& \times\left(\lambda_{1}+\lambda_{2}\right)^{m} e^{-\left(\lambda_{1}+\lambda_{2}\right) \sum_{i=1}^{m}\left(R_{i}+1\right) x_{i: m: n}}\left(\frac{\lambda_{1}}{\lambda_{1}+\lambda_{2}}\right)^{n_{1}}\left(\frac{\lambda_{2}}{\lambda_{1}+\lambda_{2}}\right)^{n_{2}} .
\end{aligned}
$$

Here, $n_{1}$ and $n_{2}$ are the number of failures due to cause 1 and cause 2 , respectively. Taking the logarithm of (3.2), and equating the partial derivatives to zero, we obtain the maximum likelihood estimators (MLE's) of $\lambda_{1}$ and $\lambda_{2}$ as

$$
\hat{\lambda}_{1}=\frac{n_{1}}{\sum_{i=1}^{m}\left(R_{i}+1\right) X_{i: m: n}} \quad \text { and } \quad \hat{\lambda}_{2}=\frac{n_{2}}{\sum_{i=1}^{m}\left(R_{i}+1\right) X_{i: m: n}}=\frac{m}{Z}-\hat{\lambda}_{1},
$$

where $Z=\sum_{i=1}^{m}\left(R_{i}+1\right) X_{i: m: n}$ denotes the total time on test. We will suppress the use of the subscript $m$ in $X_{i: m: n}$ for the rest of the chapter without causing any confusion.

From the likelihood function (3.2), it is immediate that $\left(n_{1}, \sum_{i=1}^{m}\left(R_{i}+1\right) X_{i: n}\right)$ is a jointly complete sufficient statistic for $\left(\lambda_{1}, \lambda_{2}\right)$. By Lehmann-Scheffe Theorem, to construct the UMVUE's of $\lambda_{1}$ and $\lambda_{2}$, it is sufficient to find unbiased estimators that are functions of $n_{1}$ and $\sum_{i=1}^{m}\left(R_{i}+1\right) X_{i: n}$. 
To construct the UMVUE's, we consider the following transformation (see Balakrishnan and Aggarwala (2000)):

$$
\begin{aligned}
Z_{1}= & n X_{1: n} \\
Z_{2}= & \left(n-R_{1}-1\right)\left(X_{2: n}-X_{1: n}\right) \\
\vdots & \vdots \\
Z_{m}= & \left(n-R_{1}-\ldots-R_{m-1}-m+1\right)\left(X_{m: n}-X_{m-1: n}\right)
\end{aligned}
$$

The $Z_{i}$ 's are called the spacings. It can be easily seen (Balakrishnan and Aggarwala (2000)) that $Z_{i}$ 's are i.i.d. $\exp \left(\lambda_{1}+\lambda_{2}\right)$ random variables. Therefore, $\sum_{i=1}^{m}\left(R_{i}+1\right) X_{i: n}=\sum_{i=1}^{m} Z_{i}$ is distributed as a gamma $\left(m, \lambda_{1}+\lambda_{2}\right)$ random variable. Since $n_{1}$ is a $\operatorname{Bin}\left(m, \frac{\lambda_{1}}{\lambda_{1}+\lambda_{2}}\right)$, and $n_{1}$ is independent of $\sum_{i=1}^{m}\left(R_{i}+1\right) X_{i: n}$, we have for $m>1$

$$
E\left(\hat{\lambda}_{1}\right)=\frac{m \lambda_{1}}{\lambda_{1}+\lambda_{2}} \times \frac{\lambda_{1}+\lambda_{2}}{m-1}=\frac{m}{m-1} \times \lambda_{1}
$$

and

$$
E\left(\hat{\lambda}_{2}\right)=\frac{m \lambda_{2}}{\lambda_{1}+\lambda_{2}} \times \frac{\lambda_{1}+\lambda_{2}}{m-1}=\frac{m}{m-1} \times \lambda_{2} .
$$

Simple calculation yields for $m>2$,

$$
\begin{aligned}
& V\left(\hat{\lambda}_{1}\right)=\frac{\lambda_{1} m}{m-1}\left[\frac{(m-1) \lambda_{2}+m \lambda_{1}}{(m-1)(m-2)}\right], \\
& V\left(\hat{\lambda}_{2}\right)=\frac{\lambda_{2} m}{m-1}\left[\frac{(m-1) \lambda_{1}+m \lambda_{2}}{(m-1)(m-2)}\right]
\end{aligned}
$$

and

$$
\operatorname{Cov}\left(\hat{\lambda}_{1}, \hat{\lambda}_{2}\right)=-\frac{m \lambda_{1} \lambda_{2}}{(m-1)^{2}(m-2)} .
$$

From (3.5) and (3.6), it follows immediately that the UMVUE's of $\lambda_{1}$ and $\lambda_{2}$ are given by

$$
\tilde{\lambda}_{1}=\frac{m-1}{m} \hat{\lambda}_{1} \quad \text { and } \quad \tilde{\lambda}_{2}=\frac{m-1}{m} \hat{\lambda}_{2}
$$

The variance and covariance of the estimators are given by

$$
\begin{aligned}
& V\left(\tilde{\lambda}_{1}\right)=\lambda_{1} \frac{m-1}{m}\left[\frac{(m-1) \lambda_{2}+m \lambda_{1}}{(m-1)(m-2)}\right], \\
& V\left(\tilde{\lambda}_{2}\right)=\lambda_{2} \frac{m-1}{m}\left[\frac{(m-1) \lambda_{1}+m \lambda_{2}}{(m-1)(m-2)}\right],
\end{aligned}
$$


and

$$
\operatorname{Cov}\left(\tilde{\lambda}_{1}, \tilde{\lambda}_{2}\right)=-\frac{\lambda_{1} \lambda_{2}}{m(m-2)}
$$

Note that the estimators are always negatively correlated which is evident from (3.3) as well. To estimate the survival function or the cumulative distribution function, we can use the invariance property of the MLEs. For example, the estimated survival functions due to cause 1 and cause 2 are given by

$$
\hat{\bar{F}}_{1}(t)=e^{-\hat{\lambda}_{1} t} \quad \text { and } \quad \hat{\bar{F}}_{2}(t)=e^{-\hat{\lambda}_{2} t}
$$

respectively.

Another parameter of interest in survival analysis is the relative risk rate due to a particular cause (say, cause 1). The relative risk is defined as

$$
\pi=P\left[X_{1 i}<X_{2 i}\right]=\int_{0}^{\infty} \lambda_{1} e^{-\lambda_{1} x} e^{-\lambda_{2} x} d x=\frac{\lambda_{1}}{\lambda_{1}+\lambda_{2}} .
$$

Once again, using the invariance property, the MLE of the relative risk is given by

$$
\hat{\pi}=\frac{\hat{\lambda}_{1}}{\hat{\lambda}_{1}+\hat{\lambda}_{2}}=\frac{n_{1}}{m} .
$$

For the exponential distribution in (3.1), $\lambda_{1}, \lambda_{2}$ represent the hazard rates, and $\theta_{1}=\frac{1}{\lambda_{1}}$, $\theta_{2}=\frac{1}{\lambda_{2}}$ represent the mean lifetime due to cause 1 and cause 2 , respectively. Although the MLE's and UMVUE's of $\lambda_{1}$ and $\lambda_{2}$ always exist, the UMVUE's of $\theta_{1}$ and $\theta_{2}$ do not exist. The MLE's of $\theta_{1}\left(\theta_{2}\right)$ do not exist if $n_{1}=0\left(n_{2}=0\right)$. We may then define the conditional MLE's of $\theta_{1}\left(\theta_{2}\right)$ if $n_{1}>0\left(n_{2}>0\right)$ as follows:

$$
\hat{\theta}_{1}=\frac{\sum_{i=1}^{m}\left(R_{i}+1\right) X_{i: n}}{n_{1}} \quad \text { if } \quad n_{1}>0 \quad \text { and } \quad \hat{\theta}_{2}=\frac{\sum_{i=1}^{m}\left(R_{i}+1\right) X_{i: n}}{n_{2}} \quad \text { if } n_{2}>0 .
$$

Using the forms of the estimators given above, we will now derive the exact distribution of $\hat{\lambda}_{1}, \hat{\lambda}_{2}, \hat{\theta}_{1}$ and $\hat{\theta}_{2}$. These distributions will naturally be useful in constructing confidence intervals. The cumulative distribution function of $\hat{\lambda}_{1}$ is given by

$$
\begin{aligned}
F_{\hat{\lambda}_{1}}(x) & =P\left[\hat{\lambda}_{1} \leq x\right]=P\left[\sum_{i=1}^{m} Z_{i} \geq \frac{n_{1}}{x}\right]=\sum_{j=1}^{m} P\left[\sum_{i=1}^{m} Z_{i} \geq \frac{n_{1}}{x} \mid n_{1}=j\right] P\left(n_{1}=j\right) \\
& =\sum_{j=1}^{m}\left(\begin{array}{c}
m \\
j
\end{array}\right)\left(\frac{\lambda_{1}}{\lambda_{1}+\lambda_{2}}\right)^{j}\left(\frac{\lambda_{2}}{\lambda_{1}+\lambda_{2}}\right)^{m-j} \times P\left[\sum_{i=1}^{m} Z_{i} \geq \frac{j}{x}\right] \\
& =\sum_{j=1}^{m} \sum_{i=0}^{m-1}\left(\begin{array}{c}
m \\
j
\end{array}\right)\left(\frac{\lambda_{1}}{\lambda_{1}+\lambda_{2}}\right)^{j}\left(\frac{\lambda_{2}}{\lambda_{1}+\lambda_{2}}\right)^{m-j} \frac{e^{-\left(\lambda_{1}+\lambda_{2}\right) \frac{j}{x}}\left(\lambda_{1}+\lambda_{2}\right)^{i}\left(\frac{j}{x}\right)^{i}}{i !} .
\end{aligned}
$$


Note that the distribution function of $\hat{\lambda}_{1}$ is a mixture of a discrete and a continuous distribution. We have

$$
P\left(\hat{\lambda}_{1}=0\right)=P\left(n_{1}=0\right)=\left(\frac{\lambda_{2}}{\lambda_{1}+\lambda_{2}}\right)^{m} .
$$

For $x>0$, the density function is given by

$$
f_{\hat{\lambda}_{1}}(x)=\sum_{j=1}^{m} g_{j}(x)\left(\begin{array}{c}
m \\
j
\end{array}\right)\left(\frac{\lambda_{1}}{\lambda_{1}+\lambda_{2}}\right)^{j}\left(\frac{\lambda_{2}}{\lambda_{1}+\lambda_{2}}\right)^{m-j}
$$

where

$$
g_{i}(x)=\frac{\left(i\left(\lambda_{1}+\lambda_{2}\right)\right)^{m}}{\Gamma(m)} e^{-\frac{\left(\lambda_{1}+\lambda_{2}\right) i}{x}} \frac{1}{x^{m+1}}
$$

is the density function of the inverted gamma random variable $I\left(m, i\left(\lambda_{1}+\lambda_{2}\right)\right)$. The distribution of $\hat{\lambda}_{2}$ may be obtained in a similar manner as

$$
F_{\hat{\lambda}_{2}}(x)=P\left[\hat{\lambda}_{2} \leq x\right]=\sum_{j=1}^{m} \sum_{i=0}^{m-1}\left(\begin{array}{c}
m \\
j
\end{array}\right)\left(\frac{\lambda_{2}}{\lambda_{1}+\lambda_{2}}\right)^{j}\left(\frac{\lambda_{1}}{\lambda_{1}+\lambda_{2}}\right)^{m-j} \frac{e^{-\left(\lambda_{1}+\lambda_{2}\right) \frac{j}{x}}\left(\lambda_{1}+\lambda_{2}\right)^{i}\left(\frac{j}{x}\right)^{i}}{i !} .
$$

As before,

$$
P\left(\hat{\lambda}_{2}=0\right)=P\left(n_{2}=0\right)=\left(\frac{\lambda_{1}}{\lambda_{1}+\lambda_{2}}\right)^{m},
$$

and for $x>0$ the density function of $\hat{\lambda}_{2}$ is given by

$$
f_{\hat{\lambda}_{2}}(x)=\sum_{j=1}^{m} g_{j}(x)\left(\begin{array}{c}
m \\
j
\end{array}\right)\left(\frac{\lambda_{2}}{\lambda_{1}+\lambda_{2}}\right)^{j}\left(\frac{\lambda_{1}}{\lambda_{1}+\lambda_{2}}\right)^{m-j} .
$$

We now obtain the distribution function of the conditional MLE of $\theta_{1}$ as defined in (3.13). For brevity, we denote it by $F_{\hat{\theta}_{1}}(x)$. We have

$$
\begin{aligned}
F_{\hat{\theta}_{1}}(x) & =P\left[\hat{\theta}_{1} \leq x \mid n_{1}>0\right] \\
& =\sum_{i=1}^{m} P\left[\hat{\theta}_{1} \leq x \mid n_{1}=i, n_{1}>0\right] \times P\left[n_{1}=i \mid n_{1}>0\right] \\
& =\sum_{i=1}^{m} P\left[\sum_{j=1}^{m} \frac{Z_{j}}{i} \leq x\right] \times p_{i},
\end{aligned}
$$

where

$$
p_{i}=\left(1-q^{m}\right)^{-1} \frac{m !}{i !(m-i) !}\left(\frac{\theta_{2}}{\theta_{1}+\theta_{2}}\right)^{i}\left(\frac{\theta_{1}}{\theta_{1}+\theta_{2}}\right)^{m-i} \quad \text { and } \quad q=\frac{\theta_{1}}{\theta_{1}+\theta_{2}} \text {. }
$$


Note that $\frac{Z_{j}}{i}$ is a gamma $\left(m, i\left(\frac{1}{\theta_{1}}+\frac{1}{\theta_{2}}\right)\right)$ random variable. Therefore,

$$
F_{\hat{\theta}_{1}}(x)=\sum_{i=1}^{m}\left(1-\sum_{j=0}^{m-1} \frac{e^{-\left(\frac{i}{\theta_{1}}+\frac{i}{\theta_{2}}\right) x}\left(\frac{i}{\theta_{1}}+\frac{i}{\theta_{2}}\right)^{j}}{j !}\right) \times p_{i}=\sum_{i=1}^{m} p_{i} H_{i}(x) \text { (say). }
$$

Here, $H_{i}(x)$ is the distribution function of a gamma $\left(m, i\left(\frac{1}{\theta_{1}}+\frac{1}{\theta_{2}}\right)\right)$ random variable. The density function of the conditional MLE of $\theta_{1}$ becomes

$$
f_{\hat{\theta}_{1}}(x)=\sum_{i=1}^{m} p_{i} h_{i}(x)
$$

where $h_{i}(x)$ is the density function of a gamma $\left(m, i\left(\frac{1}{\theta_{1}}+\frac{1}{\theta_{2}}\right)\right)$ random variable. Similarly, we obtain the distribution function of the conditional MLE of $\theta_{2}$ as

$$
F_{\hat{\theta}_{2}}(x)=P\left[\hat{\theta}_{2} \leq x \mid n_{2}>0\right]=\sum_{i=1}^{m} \tilde{p}_{i} H_{i}(x),
$$

where

$$
\tilde{p}_{i}=\left(1-\tilde{q}^{m}\right)^{-1} \frac{m !}{i !(m-i) !}\left(\frac{\theta_{1}}{\theta_{1}+\theta_{2}}\right)^{i}\left(\frac{\theta_{2}}{\theta_{1}+\theta_{2}}\right)^{m-i} \quad \text { and } \quad \tilde{q}=\frac{\theta_{2}}{\theta_{1}+\theta_{2}} \text {. }
$$

The corresponding density function of the conditional MLE of $\theta_{2}$ is

$$
f_{\hat{\theta}_{2}}(x)=\sum_{i=1}^{m} \tilde{p}_{i} h_{i}(x)
$$

Using (3.19) and (3.21), we may obtain easily the moments of $\hat{\theta}_{1}$ and $\hat{\theta}_{2}$. From (3.19), we have

$$
E\left(\hat{\theta}_{1}\right)=\frac{m \theta_{1} \theta_{2}}{\theta_{1}+\theta_{2}} \sum_{i=1}^{m} \frac{p_{i}}{i} \quad \text { and } \quad E\left(\hat{\theta}_{1}^{2}\right)=\frac{m(m+1) \theta_{1}^{2} \theta_{2}^{2}}{\left(\theta_{1}+\theta_{2}\right)^{2}} \sum_{i=1}^{m} \frac{p_{i}}{i^{2}} .
$$

Similarly, we obtain from (3.21)

$$
E\left(\hat{\theta}_{2}\right)=\frac{m \theta_{1} \theta_{2}}{\theta_{1}+\theta_{2}} \sum_{i=1}^{m} \frac{\tilde{p}_{i}}{i} \quad \text { and } \quad E\left(\hat{\theta}_{2}^{2}\right)=\frac{m(m+1) \theta_{1}^{2} \theta_{2}^{2}}{\left(\theta_{1}+\theta_{2}\right)^{2}} \sum_{i=1}^{m} \frac{\tilde{p}_{i}}{i^{2}} .
$$

Note that, in the expressions above, the quantities within the summation sign denote the inverse moments of positive binomial random variables. Since exact expressions are not available, we may use tabulated values of positive binomial random variables available in Edwin and Savage (1954). Since the estimators are clearly biased, tabulated values of the biases given by Kundu and Basu (2000) may be used for bias correction. 
In several examples, the investigator may be interested in testing whether the hazard rates (mean lifetimes) of the two causes are identical. For testing $H_{0}: \lambda_{1}=\lambda_{2}$, we may derive the likelihood ratio test. However, in the case of the competing risks model, the hypothesis $H_{0}$ is equivalent to testing whether the proportion of deaths due to the 2 causes are identical. This reduces to a simple test of a binomial proportion $H_{0}^{*}: p=1 / 2$. We may derive UMP or UMPU tests to determine whether there is indeed a difference in the lifetime distributions. For more than two causes of failure, we may derive tests based on the multinomial distribution.

\section{CONFIDENCE INTERVALS}

In this section, we propose four different methods of constructing confidence intervals for $\lambda_{1}, \lambda_{2}, \theta_{1}$ and $\theta_{2}$. The first method is based on the exact distributions of the MLE's derived in the previous section. The second method uses the asymptotic distributions of the estimators to obtain confidence intervals of the different parameters of interest. Finally, we construct parametric bootstrap confidence intervals using the percentile method and the bootstrap-t.

\subsection{Approximate Confidence Intervals}

In this section, we will outline the procedure for constructing approximate confidence intervals for $\lambda_{1}$ when $\lambda_{2}$ is assumed to be known. A similar construction will generate intervals for the remaining parameters. In order to use this procedure, we need to assume

that $P_{\lambda_{1}}\left[\hat{\lambda}_{1} \geq c\right]$ is monotonically increasing in $\lambda_{1}$. This assumption allows the invertibility of the pivotal quantity. This approach has been used by several authors, including Chen and Bhattacharya (1988), Gupta and Kundu (1998), Kundu and Basu (2000), and Childs et al. (2003).

Let $c(\lambda)$ be a function such that $P_{\lambda_{1}}\left[\hat{\lambda}_{1} \geq c\left(\lambda_{1}\right)\right]=\frac{\alpha}{2}$. Then, for $\lambda_{1}<\lambda_{1}^{\prime}$, we have

$$
P_{\lambda_{1}^{\prime}}\left[\hat{\lambda}_{1} \geq c\left(\lambda_{1}^{\prime}\right)\right]=P_{\lambda_{1}}\left[\hat{\lambda}_{1} \geq c\left(\lambda_{1}\right)\right] \leq P_{\lambda_{1}^{\prime}}\left[\hat{\lambda}_{1} \geq c\left(\lambda_{1}\right)\right]=\frac{\alpha}{2}
$$

From equation (4.1), we have $c\left(\lambda_{1}\right)<c\left(\lambda_{1}^{\prime}\right)$, which implies $c(\lambda)$ is an increasing function of $\lambda$. Therefore, $c^{-1}(\lambda)$ exists and is also an increasing function of $\lambda$. From (4.1), we see that

$$
P_{\lambda_{1}}\left[c^{-1}\left(\hat{\lambda}_{1}\right) \leq \lambda_{1}\right]=1-\frac{\alpha}{2},
$$


and therefore, $\lambda_{L}=c^{-1}\left(\hat{\lambda}_{1}\right)$ is the lower bound of the $100(1-\alpha) \%$ confidence interval of $\lambda_{1}$.

Similarly, we can obtain $\lambda_{U}=d^{-1}\left(\hat{\lambda}_{1}\right)$, where $d^{-1}($.$) is the inverse of the function d($. obtained as the solution of the equation

$$
P_{\lambda_{1}}\left[\hat{\lambda}_{1} \leq d\left(\lambda_{1}\right)\right]=\frac{\alpha}{2}
$$

Then, $\left(\lambda_{L}, \lambda_{U}\right)$ is a $100(1-\alpha) \%$ confidence interval for $\lambda_{1}$. Since it is not possible to obtain closed form expressions of $c($.$) and d($.$) , we need to use numerical iterative techniques to$ compute these limits. Since the confidence interval for $\lambda_{1}$ involves $\lambda_{2}$, which is unknown, we replace $\lambda_{2}$ by its MLE. To obtain the upper and lower confidence limits, we solve the following two non-linear equations:

$$
\begin{gathered}
1-\frac{\alpha}{2}=\sum_{j=1}^{m} \sum_{i=0}^{m-1}\left(\begin{array}{c}
m \\
j
\end{array}\right)\left(\frac{\lambda_{L}}{\lambda_{L}+\hat{\lambda}_{2}}\right)^{j}\left(\frac{\hat{\lambda}_{2}}{\lambda_{L}+\hat{\lambda}_{2}}\right)^{m-j} \frac{e^{-\left(\lambda_{L}+\hat{\lambda}_{2}\right) \frac{j}{\hat{\lambda}_{1}}}\left(\lambda_{L}+\hat{\lambda}_{2}\right)^{i}\left(\frac{j}{\hat{\lambda}_{1}}\right)^{i}}{i !} \\
\frac{\alpha}{2}=\sum_{j=1}^{m} \sum_{i=0}^{m-1}\left(\begin{array}{c}
m \\
j
\end{array}\right)\left(\frac{\lambda_{U}}{\lambda_{U}+\hat{\lambda}_{2}}\right)^{j}\left(\frac{\hat{\lambda}_{2}}{\lambda_{U}+\hat{\lambda}_{2}}\right)^{m-j} \frac{e^{-\left(\lambda_{U}+\hat{\lambda}_{2}\right) \frac{j}{\lambda_{1}}}\left(\lambda_{U}+\hat{\lambda}_{2}\right)^{i}\left(\frac{j}{\hat{\lambda}_{1}}\right)^{i}}{i !}
\end{gathered}
$$

The procedure outlined above requires the assumption that $P_{\lambda_{1}}\left[\hat{\lambda}_{1} \geq c\right]$ is increasing in $\lambda_{1}$. The complicated structure of the function makes it extremely difficult to provide a rigorous proof of this assumption. Numerical studies show that the result is true, and some heuristic justification along the lines of Chen and Bhattacharya (1988) or Kundu and Basu (2000) may be provided.

\subsection{Fisher Information Matrix}

In this subsection, we present the Fisher Information matrix of $\lambda_{1}$ and $\lambda_{2}$. Let $\mathbf{I}\left(\lambda_{1}, \lambda_{2}\right)$ $=\left(\left(I_{i j}\left(\lambda_{1}, \lambda_{2}\right)\right), i, j=1,2\right.$, denote the Fisher Information matrix of the parameters $\lambda_{1}$ and $\lambda_{2}$, where

$$
I_{i j}\left(\lambda_{1}, \lambda_{2}\right)=-E\left(\frac{\partial^{2} \ln \left(L\left(\lambda_{1}, \lambda_{2}\right)\right)}{\partial \lambda_{i} \partial \lambda_{j}}\right) .
$$

We have

$$
\begin{aligned}
I_{11}\left(\lambda_{1}, \lambda_{2}\right) & =\frac{m}{\lambda_{1}\left(\lambda_{1}+\lambda_{2}\right)}, \\
I_{12}\left(\lambda_{1}, \lambda_{2}\right) & =I_{21}\left(\lambda_{1}, \lambda_{2}\right)=0 \\
I_{22}\left(\lambda_{1}, \lambda_{2}\right) & =\frac{m}{\lambda_{2}\left(\lambda_{1}+\lambda_{2}\right)} .
\end{aligned}
$$


The Fisher Information matrix of $\theta_{1}$ and $\theta_{2}$, say $\mathbf{I}\left(\theta_{1}, \theta_{2}\right)$, may be obtained from $\mathbf{I}\left(\lambda_{1}, \lambda_{2}\right)$. Let $\mathbf{I}\left(\theta_{1}, \theta_{2}\right)=\left(\left(I_{i j}\left(\theta_{1}, \theta_{2}\right)\right)\right), i, j=1,2$, denote the Fisher Information matrix. Then,

$$
\begin{aligned}
I_{11}\left(\theta_{1}, \theta_{2}\right) & =\frac{m \theta_{2}}{\theta_{1}^{2}\left(\theta_{1}+\theta_{2}\right)}, \\
I_{12}\left(\theta_{1}, \theta_{2}\right) & =I_{21}\left(\theta_{1}, \theta_{2}\right)=0 \\
I_{22}\left(\theta_{1}, \theta_{2}\right) & =\frac{m \theta_{1}}{\theta_{2}^{2}\left(\theta_{1}+\theta_{2}\right)} .
\end{aligned}
$$

Using the asymptotic normality of the MLE and the above information matrix, we obtain the $100(1-\alpha) \%$ confidence intervals for $\lambda_{1}, \lambda_{2}, \theta_{1}$ and $\theta_{2}$ as

$$
\begin{aligned}
& \hat{\lambda}_{1} \pm z_{\frac{\alpha}{2}} \sqrt{\frac{\hat{\lambda}_{1}\left(\hat{\lambda}_{1}+\hat{\lambda}_{2}\right)}{m}}, \\
& \hat{\lambda}_{2} \pm z_{\frac{\alpha}{2}} \sqrt{\frac{\hat{\lambda}_{2}\left(\hat{\lambda}_{1}+\hat{\lambda}_{2}\right)}{m}} \\
& \hat{\theta}_{1} \pm z_{\frac{\alpha}{2}} \sqrt{\frac{\hat{\theta}_{1}^{2}\left(\hat{\theta}_{1}+\hat{\theta}_{2}\right)}{m \hat{\theta}_{2}}} \\
& \hat{\theta}_{2} \pm z_{\frac{\alpha}{2}} \sqrt{\frac{\hat{\theta}_{2}^{2}\left(\hat{\theta}_{1}+\hat{\theta}_{2}\right)}{m \hat{\theta}_{1}}}
\end{aligned}
$$

respectively. Here, $z_{\frac{\alpha}{2}}$ is the upper $\frac{\alpha}{2}^{t h}$ percentile point of a standard normal distribution.

\subsection{Bootstrap Confidence Intervals}

In this subsection, we construct confidence intervals based on the parametric bootstrap. The two methods that are widely used are (1) the percentile bootstrap method proposed by Efron (1982), and (2) the bootstrap-t method proposed by Hall (1988). We illustrate the procedure for the parameter $\lambda_{1}$. Intervals for the other parameters may be constructed in an analogous manner.

To obtain the percentile bootstrap confidence interval for $\lambda_{1}$, we use the following algorithm:

[1] Determine $\hat{\lambda}_{1}$ and $\hat{\lambda}_{2}$ from the sample $\left\{\left(x_{1: n}, \delta_{1}, R_{1}\right) \ldots,\left(x_{m: n}, \delta_{m}, R_{m}\right)\right\}$ using (3.3). 
[2] Generate a random variable from the gamma $\left(m, \hat{\lambda}_{1}+\hat{\lambda}_{2}\right)$ distribution, and an independent $\operatorname{Bin}\left(m, \frac{\hat{\lambda}_{1}}{\hat{\lambda}_{1}+\hat{\lambda}_{2}}\right)$ random variable. The ratio of the two random variables provides the bootstrap estimate of $\lambda_{1}$, say $\hat{\lambda}_{1}^{*}$.

[3] Repeat Step 2 NBOOT times.

[4] Let $C \hat{D} F(\mathrm{x})=P_{*}\left(\hat{\lambda}_{1}^{*} \leq x\right)$ be the cumulative distribution function of $\hat{\lambda}_{1}^{*}$. Define $\hat{\theta}_{\text {boot }}(\mathrm{x})=C \hat{D} F^{-1}(\mathrm{x})$ for a given $x$. The approximate $100(1-\alpha) \%$ confidence interval for $\lambda_{1}$ is given by

$$
\left(\hat{\theta}_{\text {boot }}\left(\frac{\alpha}{2}\right), \hat{\theta}_{\text {boot }}\left(1-\frac{\alpha}{2}\right)\right)
$$

To obtain the bootstrap-t confidence interval for $\lambda_{1}$, we use the following algorithm:

[1] Determine $\hat{\lambda}_{1}$ and $\hat{\lambda}_{2}$ from the sample $\left\{\left(x_{1: n}, \delta_{1}, R_{1}\right) \ldots,\left(x_{m: n}, \delta_{m}, R_{m}\right)\right\}$ using (3.3).

[2] Generate a random variable from the gamma $\left(m, \hat{\lambda}_{1}+\hat{\lambda}_{2}\right)$ distribution, and an independent $\operatorname{Bin}\left(m, \frac{\hat{\lambda}_{1}}{\hat{\lambda}_{1}+\hat{\lambda}_{2}}\right)$ random variable. The ratio of the two random variables provides the bootstrap estimate of $\lambda_{1}$, say $\hat{\lambda}_{1}^{*}$. Compute the variance of $\hat{\lambda}_{1}^{*}$, say $\mathrm{V}\left(\hat{\lambda}_{1}^{*}\right)$, using (3.7).

[3] Determine the $T$ statistic

$$
T^{*}=\frac{\sqrt{m}\left(\hat{\lambda}_{1}^{*}-\hat{\lambda}_{1}\right)}{V\left(\hat{\lambda}_{1}^{*}\right)} .
$$

[4] Repeat Steps 2-3 NBOOT times.

[5] From the NBOOT $T^{*}$ values obtained, determine the upper bound and the lower bound of the $100(1-\alpha) \%$ confidence bound of $\lambda_{1}$ as follows: Let $C \hat{D} F(\mathrm{x})=P_{*}\left(T^{*} \leq x\right)$ be the cumulative distribution function of $T^{*}$. For a given $x$, define

$$
\hat{\lambda}_{\text {boot }_{t}}(x)=\hat{\lambda}_{1}+m^{-\frac{1}{2}} \sqrt{V\left(\hat{\lambda}_{1}\right)} C \hat{D} F^{-1}(x) .
$$

The approximate $100(1-\alpha) \%$ bootstrap-t confidence interval for $\lambda_{1}$ is then given by

$$
\left(\hat{\lambda}_{\text {boot }_{t}}\left(\frac{\alpha}{2}\right), \hat{\lambda}_{\text {boot }_{t}}\left(1-\frac{\alpha}{2}\right)\right) .
$$

We illustrate the performance of these different methods using a simulation study and a real dataset in Sections 6 and 7, respectively. 


\section{BAYESIAN ANALYSIS}

In this section, we approach the problem from a Bayesian perspective. We first consider procedures for estimating $\left(\lambda_{1}, \lambda_{2}\right)$, followed by procedures for $\left(\theta_{1}, \theta_{2}\right)$. In the context of exponential lifetimes, $\lambda_{1}$ and $\lambda_{2}$ may be reasonably modelled using gamma priors. We assume $\lambda_{1}$ and $\lambda_{2}$ are independently distributed with gamma $\left(a_{1}, b_{1}\right)$ and gamma $\left(a_{2}, b_{2}\right)$ priors, respectively. The parameters $a_{1}, b_{1}, a_{2}$ and $b_{2}$ are all assumed to be positive. When $a_{1}=b_{1}=0$ $\left(a_{2}=b_{2}=0\right)$, we obtain non-informative priors of $\lambda_{1}\left(\lambda_{2}\right)$. The posterior density of $\lambda_{1}$ and $\lambda_{2}$ based on the gamma priors is given by

$$
l\left(\lambda_{1}, \lambda_{2} \mid \boldsymbol{x}\right)=k \lambda_{1}^{a_{1}+n_{1}-1} e^{-\lambda_{1}\left(b_{1}+\sum_{i=1}^{m}\left(R_{i}+1\right) x_{i: n}\right)} \times \lambda_{2}^{a_{2}+n_{2}-1} e^{-\lambda_{2}\left(b_{2}+\sum_{i=1}^{m}\left(R_{i}+1\right) x_{i: n}\right) .}
$$

Here, $k$ is the normalizing constant that ensures $l\left(\lambda_{1}, \lambda_{2} \mid \boldsymbol{x}\right)$ is a proper density function.

From (5.1), it is clear that the posterior density functions of $\lambda_{1}$ and $\lambda_{2}$, say $l\left(\lambda_{1} \mid \boldsymbol{x}\right)$ and $l\left(\lambda_{2} \mid \boldsymbol{x}\right)$ respectively, are independent. Further, $l\left(\lambda_{1} \mid \boldsymbol{x}\right)$ is the density function of a gamma $\left(a_{1}+n_{1}, b_{1}+\sum_{i=1}^{m}\left(R_{i}+1\right) x_{i: n}\right)$ random variable, and $l\left(\lambda_{2} \mid \boldsymbol{x}\right)$ is the density function of a gamma $\left(a_{2}+n_{2}, b_{2}+\sum_{i=1}^{m}\left(R_{i}+1\right) x_{i: n}\right)$ random variable.

The Bayes estimates of $\lambda_{1}$ and $\lambda_{2}$ under squared error loss are

$$
\hat{\lambda}_{1 \text { Bayes }}=\frac{a_{1}+n_{1}}{b_{1}+\sum_{i=1}^{m}\left(R_{i}+1\right) x_{i: n}} \quad \text { and } \quad \hat{\lambda}_{2 \text { Bayes }}=\frac{a_{2}+n_{2}}{b_{2}+\sum_{i=1}^{m}\left(R_{i}+1\right) x_{i: n}},
$$

respectively. For the non-informative priors $\left(a_{1}=b_{1}=0, a_{2}=b_{2}=0\right)$, the Bayes estimators coincide with the MLE's.

The credible intervals for $\lambda_{1}$ and $\lambda_{2}$ are obtained easily from the posterior distributions. We observe that a posteriori, $W_{1}=2 \lambda_{1}\left(b_{1}+\sum_{i=1}^{m}\left(R_{i}+1\right) x_{i: n}\right)$ and $W_{2}=2 \lambda_{2}\left(b_{2}+\sum_{i=1}^{m}\left(R_{i}+1\right) x_{i: n}\right)$ follow $\chi_{2\left(a_{1}+n_{1}\right)}^{2}$ and $\chi_{2\left(a_{2}+n_{2}\right)}^{2}$ distributions, respectively. Consequently, the $100(1-\alpha) \%$ credible interval for $\lambda_{1}$ is

$$
\left[\frac{\chi_{2\left(a_{1}+n_{1}\right), \frac{\alpha}{2}}^{2}}{2\left(b_{1}+\sum_{i=1}^{m}\left(R_{i}+1\right) x_{i: n}\right)}, \quad \frac{\chi_{2\left(a_{1}+n_{1}\right), 1-\frac{\alpha}{2}}^{2}}{2\left(b_{1}+\sum_{i=1}^{m}\left(R_{i}+1\right) x_{i: n}\right)}\right]
$$

for $a_{1}+n_{1}>0$. Similarly, when $a_{2}+n_{2}>0$, the credible interval for $\lambda_{2}$ is

$$
\left[\begin{array}{cc}
\chi_{2\left(a_{2}+n_{2}\right), \frac{\alpha}{2}}^{2} & \frac{\chi_{2\left(a_{2}+n_{2}\right), 1-\frac{\alpha}{2}}^{2}}{2\left(b_{2}+\sum_{i=1}^{m}\left(R_{i}+1\right) x_{i: n}\right)}, \\
2\left(b_{2}+\sum_{i=1}^{m}\left(R_{i}+1\right) x_{i: n}\right)
\end{array}\right] .
$$


Here $\chi_{k, \alpha}^{2}$ is the lower $\alpha^{t h}$ percentile point of the central $\chi^{2}$ distribution with $k$ degrees of freedom.

Next, we consider the Bayes estimators for $\theta_{1}$ and $\theta_{2}$. Using the assumption of gamma priors, and squared error loss, the Bayes estimators of $\theta_{1}$ and $\theta_{2}$ are

$$
\hat{\theta}_{1 \text { Bayes }}=\frac{b_{1}+\sum_{i=1}^{m}\left(R_{i}+1\right) x_{i: n}}{a_{1}+n_{1}-1} \text { and } \quad \hat{\theta}_{2 \text { Bayes }}=\frac{b_{2}+\sum_{i=1}^{m}\left(R_{i}+1\right) x_{i: n}}{a_{2}+n_{2}-1},
$$

respectively. An argument similar to the one outlined above may be used to obtain the $100(1-\alpha) \%$ credible intervals for $\theta_{1}$ and $\theta_{2}$ as

$$
\left[\frac{2\left(b_{1}+\sum_{i=1}^{m}\left(R_{i}+1\right) x_{i: n}\right)}{\chi_{2\left(a_{1}+n_{1}\right), 1-\frac{\alpha}{2}}^{2}}, \quad \frac{2\left(b_{1}+\sum_{i=1}^{m}\left(R_{i}+1\right) x_{i: n}\right)}{\chi_{2\left(a_{1}+n_{1}\right), \frac{\alpha}{2}}^{2}}\right]
$$

and

$$
\left[\frac{2\left(b_{2}+\sum_{i=1}^{m}\left(R_{i}+1\right) x_{i: n}\right)}{\chi_{2\left(a_{2}+n_{2}\right), 1-\frac{\alpha}{2}}^{2}}, \quad \frac{2\left(b_{2}+\sum_{i=1}^{m}\left(R_{i}+1\right) x_{i: n}\right)}{\chi_{2\left(a_{2}+n_{2}\right), \frac{\alpha}{2}}^{2}}\right],
$$

respectively.

In the next section, we illustrate the methods outlined here using an example.

\section{SIMULATION STUDY}

To compare the coverage probabilities and the lengths of the different confidence and credible intervals, we conducted a small simulation study. It is evident that the performance of the different methods will not depend on the censoring scheme. This has also been observed by Balakrishnan and Aggarwala (2000). The results for different $n$ and $m$ are presented in Tables 1-4. The results are based on an average over 1000 replications.

From the tables, we observe that all the procedures provide satisfactory coverage (levels close to the nominal value of $95 \%$ ). As $m$ increases, the length of the intervals decrease. Surprisingly, the intervals based on the asymptotic distribution perform well even for small sample sizes. All the procedures give similar results: however, the credible intervals based on a non-informative prior have the shortest length. Based on this study, any one of the procedures outlined in Sections 4 and 5 may be used in practice.

\section{NUMERICAL EXAMPLE}


In this section, we consider a dataset originally analyzed by Hoel (1972). The data arose from a laboratory experiment in which male mice received a radiation dose of 300 roentgens at 5 to 6 weeks of age. The cause of death for each mouse was determined by autopsy to be thymic lymphoma, reticulum cell sarcoma, or other causes. For the purpose of analysis, we consider reticulum cell sarcoma as cause 1 and combine the other 2 causes of death as cause 2. There were $n=77$ observations in the data. We generated a progressively Type-II censored sample from the original measurements with $m=25$ and censoring scheme $R_{1}=R_{2}=\ldots=R_{24}=2, R_{25}=4$. There were $n_{1}=7$ deaths due to cause 1 and $n_{2}=18$ deaths due to cause 2. Progressive censoring in these kinds of experiments may be invaluable in obtaining information on growths of tumors in the mice. At the time of death of a particular mouse, other mice may be randomly selected and removed from the study. Autopsies on these mice may lead to information on the progression of the cancer over time.

The progressively Type-II censored sample thus obtained is $(40,2),(42,2),(62,2),(163$, 2), (179, 2), (206, 2), (222, 2), (228, 2), (252, 2), (259, 2), (318, 1), (385, 2), (407, 2), (420, 2), (462, 2), (517, 2), (517, 2), (524, 2), (525, 1), (558, 1),(536, 1), (605, 1), (612, 1), (620, $2),(621,1)$.

From the above data, we obtain the following:

$$
\sum_{i=1}^{25}\left(R_{i}+1\right) x_{i: n}=28611.00
$$

which yields

$$
\begin{aligned}
& \hat{\lambda_{1}}=\frac{7}{28611}=0.000245, \quad \hat{\lambda_{2}}=\frac{18}{28611}=0.000629, \\
& \tilde{\lambda_{1}}=0.000235, \quad \tilde{\lambda_{2}}=0.000604, \\
& \operatorname{Var}\left(\hat{\lambda_{1}}\right)=9.8112 \times 10^{-9}, \quad \operatorname{Var}\left(\hat{\lambda_{2}}\right)=2.5644 \times 10^{-8}, \\
& \operatorname{Cov}\left(\hat{\lambda_{1}}, \hat{\lambda_{2}}\right)=-2.908 \times 10^{-10}, \\
& \operatorname{Var}\left(\tilde{\lambda_{1}}\right)=9.0426 \times 10^{-9}, \quad \operatorname{Var}\left(\tilde{\lambda_{2}}\right)=2.3634 \times 10^{-8}, \\
& \operatorname{Cov}\left(\tilde{\lambda_{1}}, \tilde{\lambda_{2}}\right)=-2.469 \times 10^{-10} .
\end{aligned}
$$

The relative risk due to cause 1 is

$$
\hat{\pi}=\frac{7}{25}=0.28 .
$$

The MLE's of the mean lifetimes due to cause 1 and cause 2 are given by

$$
\hat{\theta_{1}}=4087.29, \quad \hat{\theta_{2}}=1589.5 .
$$


It is quite clear that cause 2 is more severe, with lifetimes almost one-third of that of cause 1.

To assess the performance of these estimators, we construct $95 \%$ confidence and credible intervals using all the different methods outlined in Sections 4 and 5 . The results are presented in Tables 5 and 6 . The intervals are very similar for $\lambda_{1}$ and $\lambda_{2}$, in terms of the length. However, the credible intervals for $\theta_{1}$ and $\theta_{2}$ based on the non-informative prior are much wider.

\section{SOME GENERALIZATIONS AND EXTENSIONS}

\subsection{Unknown Causes of Failure}

In all the procedures outlined above, the cause of failure for all individuals in the study were assumed to be known. The problem of unknown causes of failure was originally considered by Dinse (1982) and Miyakawa (1982), and more recently by Kundu and Basu (2000). Assume we have data of type (2.2), and $k$ causes of failure are unknown. Let $I_{1}=\left\{\delta_{i} ; \delta_{i}=1\right\}$, $I_{2}=\left\{\delta_{i} ; \delta_{i}=2\right\}, I_{3}=\left\{\delta_{i} ; \delta_{i}=3\right\},\left|I_{1}\right|=n_{1},\left|I_{2}\right|=n_{2}$ and $\left|I_{3}\right|=k=m-m^{*}$ (say). Let us also assume that $m^{*}=n_{1}+n_{2}$ is fixed. The likelihood function of the observed data (2.2) is

$$
L\left(\lambda_{1}, \lambda_{2}\right)=C \times \lambda_{1}^{n_{1}} \lambda_{2}^{n_{2}}\left(\lambda_{1}+\lambda_{2}\right)^{m-m^{*}} \times e^{-\left(\lambda_{1}+\lambda_{2}\right) \sum_{i=1}^{m}\left(R_{i}+1\right) x_{i: n}}
$$

where $C=n\left(n-R_{1}-1\right) \ldots\left(n-R_{1}-\ldots-R_{m-1}-m+1\right)$ is the normalizing constant. Taking the logarithm of (7.1), and equating the partial derivatives to zeros, we obtain the MLE's of $\lambda_{1}$ and $\lambda_{2}$ as

$$
\hat{\lambda}_{1}=\left[\frac{m}{m^{*}}\right] \frac{n_{1}}{\sum_{i=1}^{m}\left(R_{i}+1\right) x_{i: n}} \quad \text { and } \quad \hat{\lambda}_{2}=\left[\frac{m}{m^{*}}\right] \frac{n_{2}}{\sum_{i=1}^{m}\left(R_{i}+1\right) x_{i: n}} .
$$

Here $n_{1}$ is a $\operatorname{Bin}\left(m^{*}, \frac{\lambda_{1}}{\lambda_{1}+\lambda_{2}}\right)$ random variable and $n_{2}$ is $\operatorname{Bin}\left(m^{*}, \frac{\lambda_{2}}{\lambda_{1}+\lambda_{2}}\right)$ random variable. Further, $\sum_{i=1}^{m}\left(R_{i}+1\right) x_{i: n}$ is a gamma $\left(m, \lambda_{1}+\lambda_{2}\right)$ random variable which is independent of $n_{1}$ and $n_{2}$.

All the procedures discussed in Sections 3 and 4 can be easily modified to the present situation. The Bayesian analysis can also be carried out along the lines of Section 5.

\subsection{Models for the Lifetime Distribution}


The first step in generalizing the assumption of exponential lifetimes is to consider the Weibull model. All the methods outlined in Sections 3-5 can be easily adapted to Weibull lifetimes. If we consider a common shape parameter, it is easy to derive explicit expressions for the two scale parameters. Unfortunately, when estimating the shape parameter, there is no closed form solution, and numerical iterative techniques will have to be employed. The expressions for the information matrix and the Bayesian estimators will also require

iterative methods. Since there is not much insight to be gained by providing the forms of the estimators, we will not present here these expressions for brevity.

We have also considered the case of dependent causes of failure. As we mentioned in the Introduction, identifiability is a major concern. If there is anecdotal or physiological evidence that the causes of failure are dependent, we could use (among others) the bivariate exponential model suggested by Marshall and Olkin (see Kotz, Balakrishnan, and Johnson (2000)).

\section{CONCLUSIONS}

In this chapter, we have considered the competing risks model when the observed data is progressively Type-II censored. We have assumed that the lifetimes under the different causes have independent exponential distributions. We have obtained the MLE's and UMVUE's of the hazard rates and the mean lifetimes, and also derived their exact distributions. Several different procedures for constructing confidence intervals have been suggested. In addition, we have derived the Bayes estimators under suitable informative and non-informative priors. A numerical example has been provided to illustrate the methods outlined in this chapter. We have also suggested some extensions and generalizations of these models.

\section{References}

Balakrishnan, N. and Aggarwala, R. (2000), Progressive Censoring: Theory, Methods and Applications, Birkhäuser, Boston.

Boag, J. W. (1949), "Maximum likelihood estimates of the proportion of patients cured by cancer therapy", Journal of the Royal Statistical Society, Series B, vol. 11, 15-44. 
Chen, S.M. and Bhattacharya, G.K. (1988), "Exact confidence bound for an exponential parameter hybrid censoring", Communications in Statistics - Theory and Methods, vol. $16,1858-1870$.

Childs, A., Chandrasekar, B., Balakrishnan, N, and Kundu, D. (2003), "Exact likelihood inference based on Type-I and Type-II hybrid censored samples from the exponential distribution", Annals of the Institute of Statistical Mathematics (to appear).

Cohen, A. C. (1963), "Progressively censored samples in life testing", Technometrics, vol. 5, 327-329.

Cohen, A. C. (1966), "Life testing and early failure", Technometrics, vol. 8, 539-549.

Crowder, M. J. (2001), Classical Competing Risks, Chapman \& Hall, Boca Raton, Florida.

David, H.A. and Moeschberger, M.L. (1978), The Theory of Competing Risks, Griffin, London.

Dinse, G.E. (1982), "Non-parametric estimation of partially incomplete time and type of failure data", Biometrics, vol. 38, 417-431.

Edwin, G.L. and Savage, R.I. (1954), "Tables of expected value of 1/X for positive Bernoulli and Poisson variables", Journal of the American Statistical Association, vol. 49, 169-177.

Efron, B. (1982), The Jackknife, the Bootstrap and Other Resampling Plans, CBMS-NSF Regional Conference series in Applied Mathematics, vol. 38, SIAM Philadelphia, USA.

Gupta, R.D. and Kundu, D. (1998), "Hybrid censoring schemes with exponential failure distribution", Communications in Statistics - Theory and Methods, vol. 27, 3065-3083.

Hall, P. (1988), "Theoretical comparison of bootstrap confidence intervals", Annals of Statistics, vol. 16, 927-953.

Hoel, D. G. (1972), "A representation of mortality data by competing risks", Biometrics, 
vol. $28,475-488$.

Kalbfleisch, J. D. and Prentice, R. L. (1980), The Statistical Analysis of Failure Time Data, John Wiley \& Sons, New York.

Kotz, S., Balakrishnan, N. and Johnson, N. L. (2000), Continuous Multivariate Distributions, Volume 1: Models and Applications, Second edition, John Wiley \& Sons, New York.

Kundu, D. and Basu, S. (2000), "Analysis of incomplete data in presence of competing risks", Journal of Statistical Planning and Inference, vol. 87, 221-239.

Miyakawa, M. (1982), "Statistical analysis of incomplete data in competing risks model", Journal of Japanese Society Quality Control, vol. 12, 49-52.

Miyakawa, M. (1984), "Analysis of incomplete data in competing risks model", IEEE Transactions on Reliability, vol. 33, 293-296. 
Table 1: $\mathrm{n}=20, \mathrm{~m}=10, \lambda_{1}=1.0, \lambda_{2}=0.8$

\begin{tabular}{|c|c|c|c|}
\hline Methods & Parameters & Average Length & Coverage Percentage \\
\hline Bayes & $\lambda_{1}$ & 1.76078 & 0.958 \\
& $\lambda_{2}$ & 1.56975 & 0.936 \\
\hline \multirow{2}{*}{ Asymptotic } & $\lambda_{1}$ & 1.78011 & 0.937 \\
& $\lambda_{2}$ & 1.57125 & 0.922 \\
\hline Exact & $\lambda_{1}$ & 1.52690 & 0.952 \\
& $\lambda_{2}$ & 1.37600 & 0.947 \\
\hline Boot-P & $\lambda_{1}$ & 2.12155 & 0.933 \\
& $\lambda_{2}$ & 1.83684 & 0.930 \\
\hline Boot-t & $\lambda_{1}$ & 2.04192 & 0.939 \\
& $\lambda_{2}$ & 1.82465 & 0.915 \\
\hline
\end{tabular}

Table 2: $\mathrm{n}=50, \mathrm{~m}=20, \lambda_{1}=1.0, \lambda_{2}=0.8$

\begin{tabular}{|c|c|c|c|}
\hline Methods & Parameters & Average Length & Coverage Percentage \\
\hline Bayes & $\lambda_{1}$ & 1.22373 & 0.955 \\
& $\lambda_{2}$ & 1.08401 & 0.936 \\
\hline \multirow{2}{*}{ Asymptotic } & $\lambda_{1}$ & 1.23344 & 0.940 \\
& $\lambda_{2}$ & 1.09470 & 0.926 \\
\hline Exact & $\lambda_{1}$ & 1.30335 & 0.966 \\
& $\lambda_{2}$ & 1.16623 & 0.958 \\
\hline Boot-P & $\lambda_{1}$ & 1.33695 & 0.949 \\
& $\lambda_{2}$ & 1.18564 & 0.934 \\
\hline Boot-t & $\lambda_{1}$ & 1.31618 & 0.922 \\
& $\lambda_{2}$ & 1.22481 & 0.926 \\
\hline
\end{tabular}


Table 3: $\mathrm{n}=75, \mathrm{~m}=25, \lambda_{1}=1.0, \lambda_{2}=0.8$

\begin{tabular}{|c|c|c|c|}
\hline Methods & Parameters & Average Length & Coverage Percentage \\
\hline Bayes & $\lambda_{1}$ & 1.08492 & 0.946 \\
& $\lambda_{2}$ & 0.96296 & 0.932 \\
\hline \multirow{2}{*}{ Asymptotic } & $\lambda_{1}$ & 1.09183 & 0.949 \\
& $\lambda_{2}$ & 0.97073 & 0.920 \\
\hline Exact & $\lambda_{1}$ & 1.17490 & 0.951 \\
& $\lambda_{2}$ & 1.05530 & 0.956 \\
\hline Boot-P & $\lambda_{1}$ & 1.15351 & 0.931 \\
& $\lambda_{2}$ & 1.02053 & 0.936 \\
\hline Boot-t & $\lambda_{1}$ & 1.14355 & 0.921 \\
& $\lambda_{2}$ & 1.04977 & 0.929 \\
\hline
\end{tabular}

Table 4: $\mathrm{n}=75, \mathrm{~m}=35, \lambda_{1}=1.0, \lambda_{2}=0.8$

\begin{tabular}{|c|c|c|c|}
\hline Methods & Parameters & Average Length & Coverage Percentage \\
\hline Bayes & $\lambda_{1}$ & 0.90954 & 0.937 \\
& $\lambda_{2}$ & 0.80899 & 0.945 \\
\hline \multirow{2}{*}{ Asymptotic } & $\lambda_{1}$ & 0.91367 & 0.940 \\
& $\lambda_{2}$ & 0.81363 & 0.946 \\
\hline Exact & $\lambda_{1}$ & 1.00572 & 0.955 \\
& $\lambda_{2}$ & 0.93749 & 0.956 \\
\hline Boot-P & $\lambda_{1}$ & 0.93049 & 0.945 \\
& $\lambda_{2}$ & 0.84728 & 0.950 \\
\hline Boot-t & $\lambda_{1}$ & 0.93652 & 0.928 \\
& $\lambda_{2}$ & 0.86743 & 0.939 \\
\hline
\end{tabular}


Table 5: Confidence and Credible Intervals

\begin{tabular}{|l|l|l|}
\hline Method & $\lambda_{1}$ & $\lambda_{2}$ \\
\hline Exact (MLE) & $(0.000064,0.000426)$ & $(0.000338,0.000920)$ \\
\hline $\begin{array}{l}\text { Credible Interval } \\
\text { (non-informative prior) }\end{array}$ & $(0.000098,0.000456)$ & $(0.000372,0.000951)$ \\
\hline Bootstrap-t & $(0.000087,0.000448)$ & $(0.000349,0.000949)$ \\
\hline Percentile & $(0.000069,0.000460)$ & $(0.000361,0.000959)$ \\
\hline
\end{tabular}

Table 6: Confidence Intervals

\begin{tabular}{|l|l|l|}
\hline Method & $\theta_{1}$ & $\theta_{2}$ \\
\hline Exact (MLE) & $(1059.38,7115.20)$ & $(855.19,2328.81)$ \\
\hline $\begin{array}{l}\text { Credible Interval } \\
\text { (non-informative prior) }\end{array}$ & $(2192.98,10204.08)$ & $(1051.53,2688.17)$ \\
\hline
\end{tabular}

